\title{
Effects of Lithium Monotherapy for Bipolar Disorder on Gene Expression in Peripheral Lymphocytes
}

\author{
Amit Anand $^{a, b}$ Jeanette N. McClintick ${ }^{c}$ Jill Murrell ${ }^{c}$ Harish Karne ${ }^{a, b}$ \\ John I. Nurnberger ${ }^{b}$ Howard J. Edenberg ${ }^{c}$ \\ ${ }^{a}$ Center for Behavioral Health, Cleveland Clinic, Cleveland, Ohio, ${ }^{b}$ Department of Psychiatry, and ${ }^{\mathrm{c}}$ Department \\ of Biochemistry and Molecular Biology and Center for Medical Genomics, Indiana University School of Medicine, \\ Indianapolis, Ind., USA
}

\section{Key Words}

Lithium · Gene expression · Bipolar disorder · Depression . Mania - Immune genes - Interferon - Signal transduction . Pathway analysis

\begin{abstract}
Background: This study investigated the effect of lithium monotherapy on peripheral lymphocyte gene expression in bipolar disorder (BD). Method: Twenty-two medication-free bipolar subjects (11 hypomanic, 11 depressed) were started on lithium monotherapy. Closely matched healthy subjects $(n=15)$ were included as controls but did not receive treatment. Blood RNA samples were collected at baseline and after 2 and 8 weeks of treatment. RNA expression was measured using the Affymetrix GeneChip ${ }^{\circledR}$ Human Gene 1.0 ST Array followed by Ingenuity pathways analysis. The results for the contrast of weeks 2 and 8 were not significantly different and were combined. Results: In BD subjects, 56 genes showed significant (false discovery rate $<0.1$ ) expression changes from baseline; the effect sizes and directions for all of these were similar at weeks 2 and 8. Among these were immune-related genes (IL5RA, MOK, IFI6, and RFX2), purinergic receptors (P2RY14, P2RY2, and ADORA3) and signal
\end{abstract}

transduction-related genes (CAMK1 and PIK3R6). Pathway and upstream regulator analysis also revealed that lithium altered several immune- and signal transduction-related functions. Differentially expressed genes did not correlate with week 8 clinical response, but other genes involved in protein synthesis and degradation did. Conclusion: Peripheral gene expression may serve as a biomarker of lithium effect.

(c) 2016 S. Karger AG, Basel

\section{Introduction}

Lithium has been used in the treatment of bipolar disorder (BD) for nearly 6 decades, and remains one of the most effective treatments for this disorder [1]. Lithium effects are thought to be mediated through effects on neurotransmitter systems such as serotonin as well as its effects on signal transduction, e.g. the inhibition of glycogen synthase kinase- $3 \beta$ activity and inhibition of inositol monophosphatases [2].

However, the clinical observation that lithium's therapeutic effects can take many weeks to occur and that the therapeutic effectiveness on mood stabilization increases

\section{KARGER}

E-Mail karger@karger.com

www.karger.com/mnp
(C) 2016 S. Karger AG, Basel

2296-9209/16/0023-0115\$39.50/0
Amit Anand, MD

Center for Behavioral Health, Cleveland Clinic

9500 Euclid Avenue P57

Cleveland, OH 44122 (USA)

E-Mail ananda@ccf.org 
the longer and more consistently lithium is administered suggests that lithium effects may be occurring at a transcriptional level leading to changes in neuronal plasticity [3]. Indeed, recent studies have indicated significant effects of lithium on the expression of a number of genes involved in neuronal plasticity and function. Lithium has been shown to increase the expression of the antiapoptotic gene BCL2 [4] and also to increase cAMP-responsive element-/binding protein-mediated gene transcription [5]. More recently, microarray techniques have been applied to study the effects of lithium on mouse brain [6, 7], rat brain [8], and in human neuronal or peripheral blood cell lines [9-13]. These studies have reported effects of lithium on the expression of a number of genes involved in ion channel and receptor function, signal transduction (including the phosphatidylinositol system), neuroprotective mechanisms, endothelial growth factor, energy metabolism, and thyroid function.

However, as most studies have been conducted in animal models or in vitro in human cell lines, the relationship of these changes in gene expression to clinical therapeutic response to lithium remains unclear. In the past decade, gene expression in peripheral blood lymphocytes has been used as a proxy for central nervous system (CNS) gene expression [14]. As brain biopsy is unrealistic in living humans, lymphocyte gene expression provides a convenient and accessible alternative to study molecular changes in health and disease [14]. Sullivan et al. [15] demonstrated that gene expression in peripheral blood lymphocytes is reasonably well correlated with expression in the CNS. Nearly half of the candidate genes relevant to schizophrenia were expressed both in blood and in prefrontal cortex, and the expression levels of many biologically relevant classes of genes were not significantly different between blood and prefrontal cortex [15].

Beech et al. [16] conducted studies of gene expression changes in peripheral lymphocytes in bipolar subjects on lithium treatment for 1 month as well as bipolar subjects prospectively treated with lithium for 6 months [17]. After 6 months of treatment, responders had a greater upregulation of antiapoptotic genes (BCL2) while proapoptotic genes were downregulated. However, these studies were conducted while the subjects were simultaneously on a number of other medications; therefore, the results were difficult to interpret as solely due to lithium effects.

In this study, we investigated for the first time the acute ( 2 weeks) and long-term (8 weeks) effect of lithium monotherapy on peripheral gene expression in outpatient bipolar subjects who were medication free for at least 2 weeks prior to entry into the study and had not had treatment with lithium for at least 6 months. We hypothesized that lithium treatment would change the pattern of gene expression, and we highlight gene networks likely to be involved in its mechanism of action.

\section{Methods}

\section{Study Design}

Individuals with $\mathrm{BD}$ in either a depressed (BPD) or a hypomanic (BPM) state, who were medication free for at least 2 weeks and had no history of treatment with lithium for the past 6 months were recruited from an outpatient psychiatry clinic and by advertisement to the community. Psychiatrically healthy adults were recruited by advertisement. After a screening visit, the study involved baseline collection of blood sample for gene expression (RNA). Subsequently, BD subjects were treated with lithium, and repeat RNA samples were collected after 2 weeks (week 2 ) and 8 weeks (week 8) of treatment.

During the screening visit, after complete description of the study to the subjects, written informed consent, approved by the Institutional Review Board at the Indiana University School of Medicine, was obtained. Subjects underwent a detailed structured diagnostic interview: the Diagnostic Interview for Genetic Studies (DIGS) [18], as well as a clinical interview by a psychiatrist to determine the appropriate Diagnostic and Statistical Manual 4th Edition Text Revision (DSM-IV-TR) [19] diagnoses. Subjects were also rated on the 17-item Hamilton Depression Rating Scale (HDRS) and Young Mania Rating Scale (YMRS) [20] on the day of baseline collection of blood for gene expression and weekly thereafter for the 8 weeks of lithium treatment. Clinical improvement was assessed by a psychiatrist with the Clinical Global Impression of Severity (CGIS) and Improvement (CGII) Scale [21] scores at week 2 and week 8 for overall bipolar illness.

\section{Subjects}

BD patients aged 18-60 years were included in the study if they satisfied the DSM-IV-TR criteria for hypomanic (BPM) or a depressed episode (BPD). The exclusion criteria for participants included the following: lifetime diagnosis of schizophrenia or schizoaffective disorder; a current primary anxiety disorder; use of psychotropic medications in the past 2 weeks; fluoxetine use over the past 4 weeks; lithium in the past 6 months; acute suicidal or homicidal ideation or behavior; recent ( $<1$ week) or current inpatient hospitalization; a diagnosis of substance dependence within the past year, except nicotine; positive urinary toxicology screening at baseline; use of alcohol in the past 1 week; serious medical or neurological illness, and current pregnancy or breast-feeding.

Healthy subjects (18-60 years) additionally had no personal or family history of serious psychiatric or alcohol or substance abuse/ dependence as determined by the Mini International Neuropsychiatric Interview (MINI) [22] or history of serious medical illness.

\section{Lithium Treatment}

Immediately after baseline blood collection was completed, BD subjects were started on lithium treatment, $300 \mathrm{mg}$ twice daily. Levels were checked after approximately 5 days and, if necessary, lithium dose was increased to achieve trough levels between 0.5 and $1.0 \mathrm{mEq} / \mathrm{l}$ depending on efficacy and tolerance. Lithium levels 
were also checked near the time of the second and third RNA collection. Healthy subjects were not treated but had repeat RNA samples collected at the same time points as the BD subjects.

\section{RNA Samples}

Samples were collected in PaxGene tubes $(2.5 \mathrm{ml})$, transported to the laboratory at room temperature and then stored at $-20^{\circ} \mathrm{C}$ until processed using the Paxgene protocol (Qiagen). Briefly, the samples were thawed and held at room temperature for $2 \mathrm{~h}$. The samples were centrifuged and the supernatants removed. The pellets were then treated with proteinase $\mathrm{K}$ at $65^{\circ} \mathrm{C}$, then processed twice through PAXgene RNA spin columns, treated with DNase I, and processed again through PAXgene RNA spin columns. The RNA samples were stored at $-80^{\circ} \mathrm{C}$.

\section{Gene Expression Analysis}

The gene expression analysis was conducted in the Center for Medical Genomics (CMG), a core facility of the Indiana Clinical and Translational Sciences Institute. Quantity and quality of the RNA was measured using a Nanodrop spectrophotometer and Agilent Bioanalyzer. RIN (RNA integrity number) values ranged from 5.7 to 9.6, with a mean 8.3 (SD 0.67). One hundred-nanogram aliquots of each RNA were labeled and hybridized to an Affymetrix GeneChip ${ }^{\circledR}$ Human Gene 1.0 ST Array. The processing and labeling of samples was done in batches with all samples from a single individual processed together, and the individuals balanced for cases versus controls. All samples were hybridized individually. Scanned arrays were examined for defects and other quality issues.

\section{Statistical Analysis}

Data were imported into Partek Genomics Suite. Batch effects were removed from the data. Principal components analysis was used to identify outlier arrays; one was found and removed from further analysis. Analysis was done using $\log _{2}$ transformed data. Data for all BD patients for the 3 time points were analyzed using factors of time point (baseline, week 2, week 8), state (manic, depressed), ID (patient identifier), and interaction between state and time point. Probe sets with a mean $\log _{2}$ expression $<4.0$ for all groups were removed from subsequent analyses [23]. False discovery rate (FDR) was calculated using the Storey q value [24].

Contrasts were performed for the effect of treatment [week 2 vs. baseline, week 8 vs. baseline, week 8 vs. week 2 , and all treated (week 2 and 8 combined) vs. baseline] for all BD subjects combined as well as manic and depressed groups separately. The effect of time was also computed for the healthy subjects group, and healthy subjects were compared with bipolar subjects at time 0 . Genes with significantly different expression were identified at an FDR of 0.1.

For pathway analysis, the results from the contrast of week 2 and week 8 (combined) vs. baseline were imported into Qiagen Ingenuity Pathway Analysis (IPA), winter 2015 release. Core analyses were performed using genes with FDR $<0.40$; this lower threshold was used to obtain an adequate number of genes (301) for analysis. Pathways that contained fewer than 4 genes were omitted from table 2 .

We looked for a correlation of clinical response in terms of overall bipolar illness (CGIS and CGII scores) with gene expression changes in the bipolar patients at week 8 . The Spearman correlation was calculated using $\mathrm{R}$. The change in gene expression was measured by the $\log _{2}$ ratio of week 8 expression/baseline for each

Lithium Effects on Gene Expression in Bipolar Disorder individual for all genes. This expression difference was compared with CGII (final improvement measure) and the difference in CGIS as measured by CGIS at baseline - CGIS at week 8 only, as only at the end of 8 weeks of treatment can a meaningful change in mood be ascertained. We used Spearman correlation for this analysis because the CGII and CGIS scores are ordinal and expression is a continuous variable. We also examined the correlation of changes in the HDRS and YMRS scores within each group with changes in gene expression.

\section{Results}

Gene expression data from 22 BD subjects (11 BPM and $11 \mathrm{BPD}$ ) and 15 healthy subjects were included in the analysis based on the inclusion criteria. Data for 1 healthy subject were removed from the analysis as an outlier, based upon principal components analysis. For time week 2, data from 9 BPM subjects were available. Though one inclusion criterion was a medication-free period of 2 weeks, most patients were medication free for much longer periods. All patients were actually medication free for more than 8 weeks (range: $8-1,440$ weeks; mean \pm SD = $203 \pm 325$ weeks), and 2 subjects were treatment naive. Demographic and illness characteristics of the sample are presented in online supplementary table 1 (see www. karger.com/doi/10.1159/000446348 for all online suppl. material).

\section{Lithium Effects on Gene Expression}

Gene expression in the bipolar subjects at time 0 (before treatment) did not differ significantly from that in the controls, and there was no significant difference in the healthy subjects when comparing either week 2 or week 8 with baseline. Changes in gene expression were seen between time 0 and week 2 (online suppl. table 2), and between time 0 and week 8 (online suppl. table 3 ). Similar to findings at time 0 , there were no significant differences between the bipolar and healthy groups at time 2 and time 8 .

Among the bipolar subjects, initial analysis of the gene expression data showed no genes that significantly differed between weeks 2 and 8 . Therefore, data from both time points were used for further analyses to increase the power of the experiment. For the BD group as a whole, 63 transcripts that represent 56 unique genes showed a significant (FDR $<0.10)$ change in expression (table 1). The effect sizes and directions for all of these genes were similar at week 2 and week 8 . There were 37 genes with increased expression and 19 with decreased expression. 
Table 1. Change in gene expression (in order of significance) after lithium treatment ( 2 and 8 weeks combined and separately) in all bipolar subjects $(\mathrm{n}=22)$ at FDR $<0.1$

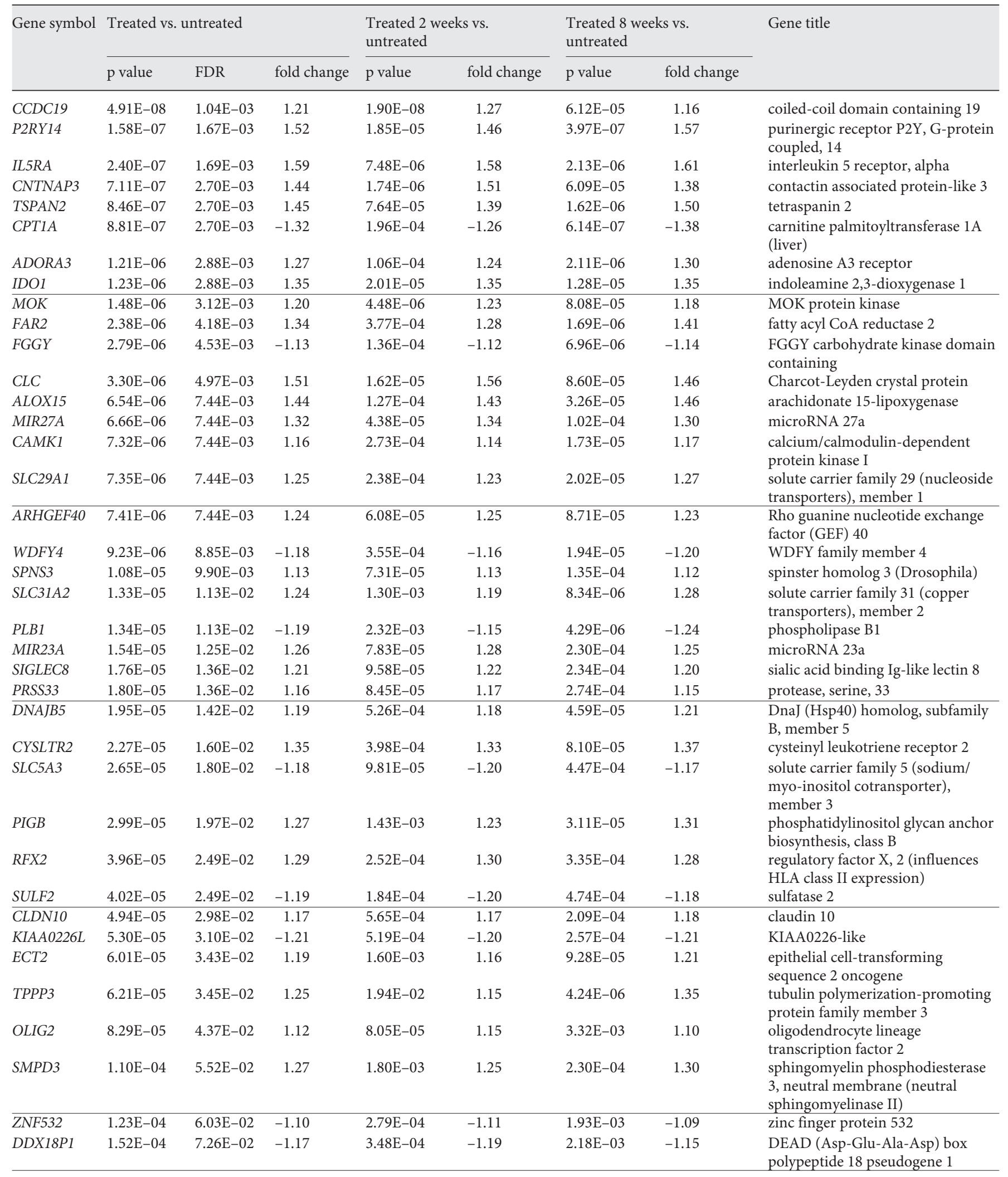


Table 1 (continued)

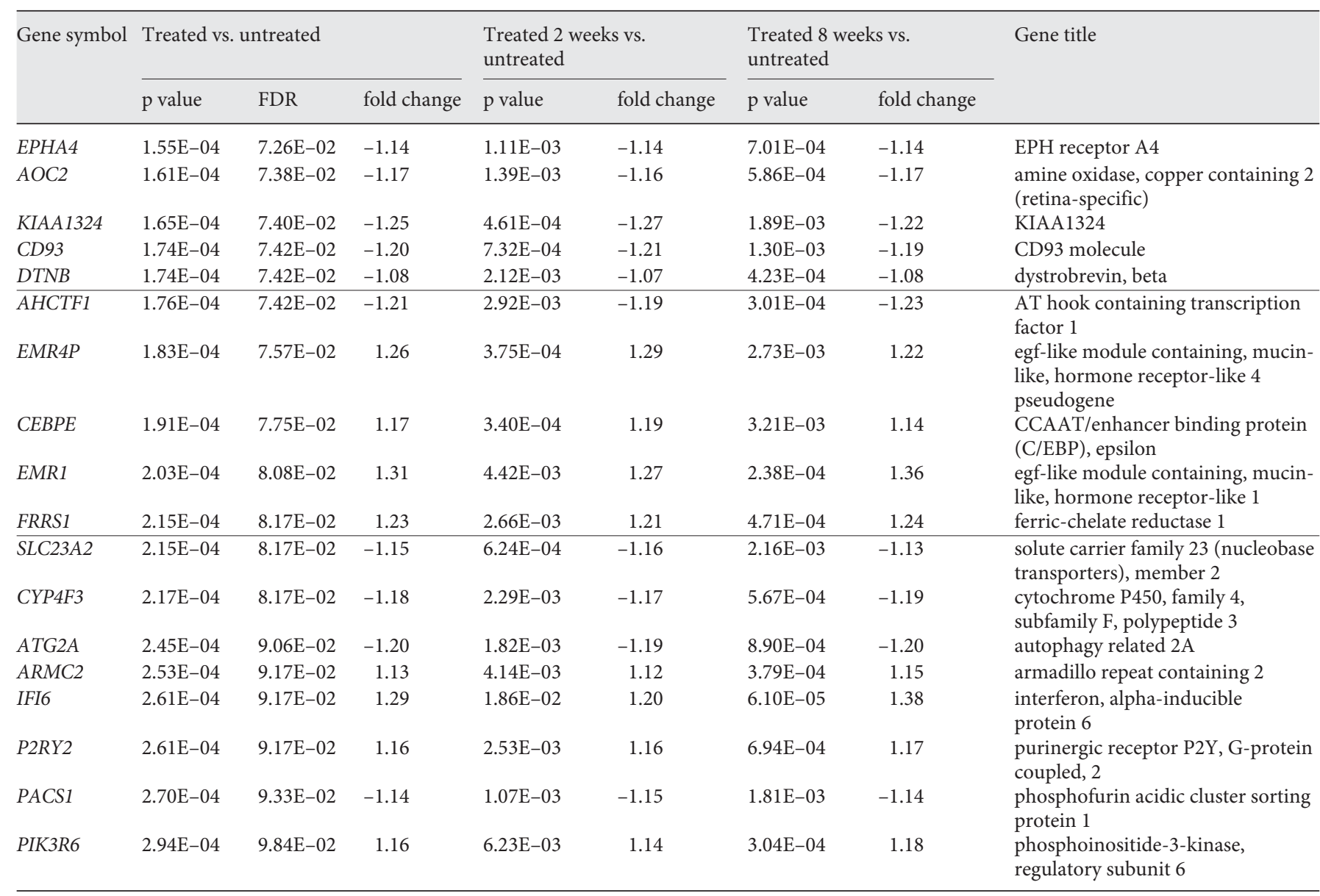

Among the genes upregulated were those related to immune function: interleukin 5 receptor (IL5RA) and interferon alpha-inducible protein IFI6, and a regulatory factor that affects HLA class II expression (RFX2). Other genes that were upregulated were several protein kinases and phosphatases related to signal transduction including CAMK1, PIK3R6, and MOK. Also upregulated were several regulatory molecules, including two purinergic receptors (P2RY14 and P2RY2) and the adenosine A3 receptor (ADORA3), a CCAAT/enhancer binding protein (CEBPE), and two microRNAs (miRNA23a, miRNA27a). Interestingly, oligodendrocyte lineage transcription factor 2 (OLIG2) was upregulated, suggesting some remodeling in the brain. A different transcription factor, AHCTF1, was downregulated, along with the ephrin receptor A4 (EPHA4).

No genes were found for which lithium had a significantly differential effect in the BPM group versus the $\mathrm{BPD}$ group, but the numbers were small, so the power to detect such differences was very limited. We conducted a visual inspection of the pattern of changes in each of the subjects for the transcripts which showed significant change. No outliers were identified. With the small number of subjects studied, only group level inferences can be made.

\section{Pathway Analysis of Lithium Effects}

To identify the pathways affected during lithium treatment, we conducted IPA using a relaxed criterion (FDR $\leq 0.40$ ) for genes which showed expression to yield sufficient genes for analysis (301 genes). This threshold was chosen as it yielded the optimum number of transcripts for pathway analysis. Thirty-eight pathways were found significant $(\mathrm{p}<0.05$; table 2$)$. These include immune-related pathways (interferon signaling, IL-3, IL-12, IL-17, and IL-17A), glucocorticoid, VDR/RXR, EGF and aldosterone receptor signaling, and PI3 kinase signaling. Two 
Table 2. IPA pathway analysis of lithium effects

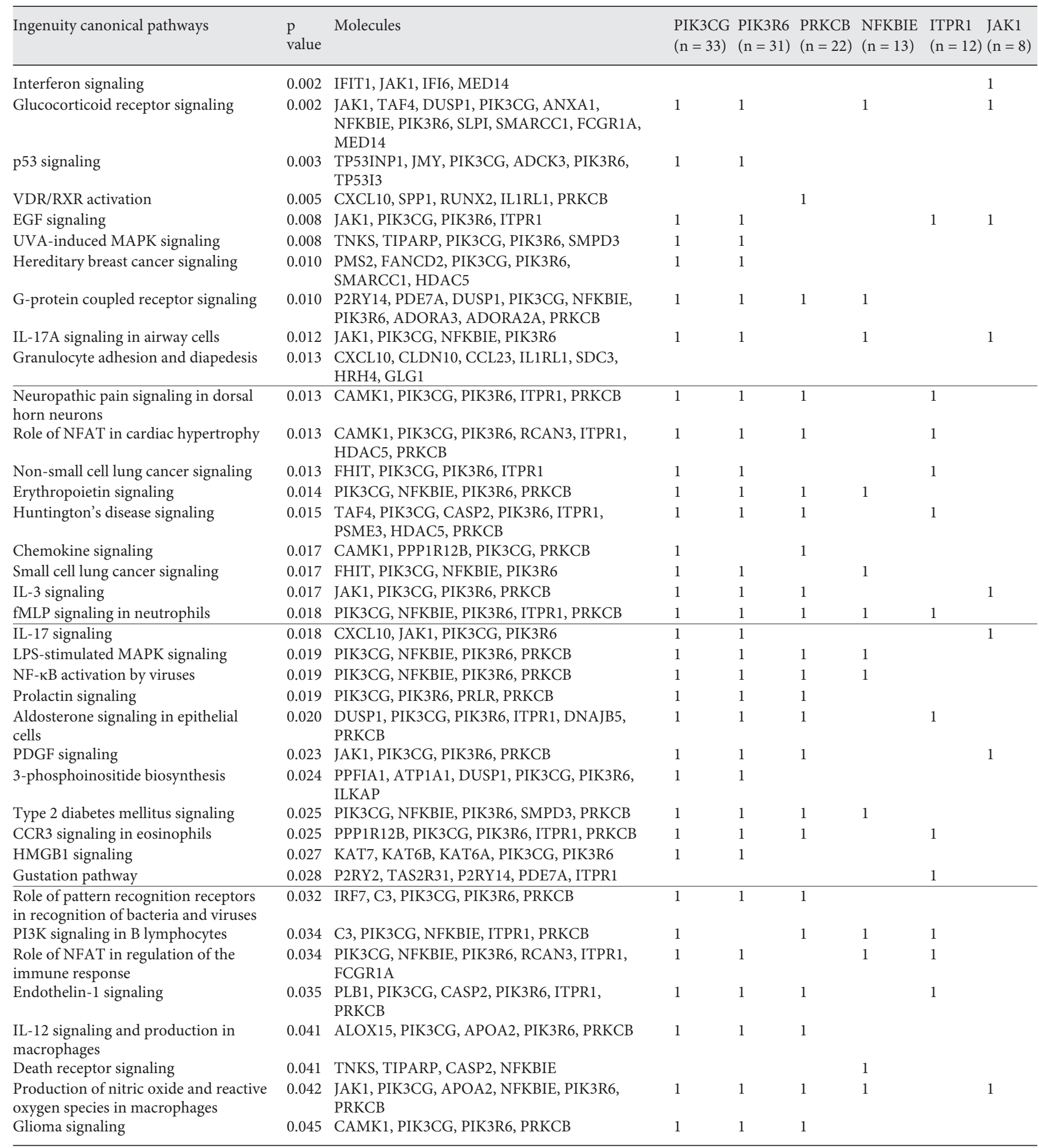

Pathways differentially expressed with lithium treatment. IPA using the 301 genes with expression changes at FDR $\leq 0.40$. Pathways are listed if $\mathrm{p}<0.05$ and they contained at least 4 genes with altered expression. 
PI3 kinase-related genes, PIK3CG and PIK3R6, were found in most of these pathways. Other genes found in many pathways were NFKBIE, ITRP1, JAK1, and CAMK1.

IPA upstream regulator analysis, which identifies molecules that can produce patterns of expression significantly related to those found (i.e. can identify what might potentially cause the changes in expression), identified many transcription factors, transmembrane receptors, cytokines, and drugs (online suppl. table 4). Eleven cytokines were identified, along with interferons, NFKB and one of its regulators IKBKE, interferon regulatory factors (IRF3, IRF5, IRF7), toll-like receptors (TLR3, TLR7, TLR9), and TNF. This suggests major changes in immune regulation. This analysis identified drugs that have effects either similar to those found (positive activation Z scores; e.g. oblimersen) or opposite to those found (negative $\mathrm{Z}$ scores; e.g. fenofibrate).

\section{Gene Expression and Pathway Correlates of}

Treatment Response

We found 491 genes for which a change in expression was significantly correlated (FDR $\leq 0.10$ ) with a change in the clinical severity of illness as measured by change in the CGIS score at week 8 (online suppl. table 5). The absolute value of the correlation ranged from 0.60 to 0.83 . Most of these genes (415) were positively correlated with improvement in CGIS. Surprisingly, only 13 of these genes were even nominally changed in expression (in the combined analysis $8.4 \times 10^{-4}<\mathrm{p}<0.050$ ). Three pathways were identified: the protein ubiquitination pathway and two related to protein synthesis, EIF2 signaling and eIF4 and p70S6K signaling. No significant change was found for the correlation with CGII scores. No significant correlations were found for changes in HDRS and YMRS scores with changes in gene expression within the BPD and BPM groups, respectively, as well as for the BP group as a whole.

\section{Discussion}

In this first study of the effect of lithium monotherapy on peripheral blood gene expression in medication-free manic and depressed subjects, we identified significant changes in transcripts related to genes and pathways involved in immune function, signal transduction, cell growth and motility, lipid metabolism and function, as well as stress response. Healthy control subjects did not show any significant changes on repeated testing at the same time points, and the untreated and treated bipolar patients did not show significant differences compared to controls. Among the bipolar subjects, changes at week 2 and week 8 did not significantly differ, indicating that the effects of lithium on gene expression in blood had plateaued by week 2 .

The effects of lithium were found to be most extensive on immune system genes, which perhaps is not surprising since this study focused on blood cells. However, other work has shown a significant correlation between gene expression in blood and brain that includes many genes related to schizophrenia [15]. There is accumulating evidence that altered immunological functioning and immune system activation may be associated with states of depression [25] as well as mania [26]; these functions also appear to be generally disrupted in the pathophysiology of $\mathrm{BD}[27,28]$. Lithium has been shown to decrease cyclooxygenase- 2 and prostaglandin activity, and this mechanism has been proposed as a contributor to its therapeutic action in BD [29]. The results of this study strengthen the evidence that lithium mechanism of action may be related to changes in genetic pathways related to immune function.

Three purinergic receptors were among the genes with altered expression: purinergic receptors P2RY14 and P2RY2, both increased by lithium, and the adenosine A3 receptor ADORA3, also increased by lithium. Purinergic receptors are G-protein-coupled receptors widely expressed in the brain, and they have been related to pain as well as neural stem cell regulation. The higher expression of oligodendrocyte lineage transcription factor 2 (OLIG2) also suggests that lithium might cause some remodeling of the brain. OLIG2 was reported to be reduced in the prefrontal cortex of patients with BD [30].

The two significantly altered microRNAs, miR23a and miR27a, are likely to play regulatory roles. miR23a is upregulated in gliomas, and antisense to this molecule reduced proliferation, migration, and invasion of glioma cell lines [31]. Glioma signaling was among the pathways significantly altered (table 2 ).

Several protein kinases and phosphatases were upregulated. PIK3R6 (phosphoinositide-3-kinase, regulatory subunit 6) was modestly upregulated, and PIK3CG (phosphoinositide-3-kinase, catalytic, gamma polypeptide) was modestly downregulated, although not meeting the FDR standard. This suggests overall reduction in PIK3 activity. PIK3s are involved in a very wide range of cellular functions, as attested by the fact that they appear in 33 of the 38 pathways that were significantly altered by lithium (table 2). The pathway of PIK3 signaling in B lym- 
phocytes was altered; C3, PIK3CG, NFKBIE, ITPR1, and PRKCB all contributed to this alteration. Six genes contributed to the alteration in 3-phosphoinositide biosynthesis. Perhaps surprisingly, glycogen synthase kinase-3 activity was not significantly altered.

Virtually all of the pathways altered involved signaling of one kind or another (table 2); as noted, changes in PIK3 subunits contribute to most of them, with specificity determined by the other transcripts that were altered. Some of these pathways involve the brain or neural cells (e.g. glioma signaling, neuropathic pain signaling, and, of course, the PIK3 pathways).

Transcripts related to lipid metabolism were upregulated (FAR2, SLC31A2) but transcripts for CPTI1 (related to mitochondrial fatty oxidation), PLB1 (related to lipid absorption), and FGGY (related to carbohydrate phosphorylation) were downregulated. Lithium efficacy has been previously reported to be associated with decreased lipid peroxidation [32].

A number of genes were correlated with change in illness severity in clinical status at week 8 , as measured by CGIS (online suppl. table 5). However, only a few of these genes showed even nominally significant changes. The patients used for this study included patients that were depressed and manic at baseline, and had a wide range of responses to lithium. Furthermore, some hypomania patients who responded to lithium in terms of hypomania became a bit depressed at the end of 8 weeks of lithium treatment. Hence, their illness severity as judged with the CGIS did not change much. Therefore, it is not too surprising that the genes that were widely affected (significant) across the whole cohort of patients were not seen in the list of genes that correlate with the response of each patient (change in CGIS). Probably, for the same reason the change in the HDRS and YMRS and CGII scores did not correlate with the changes in gene expression.

There are several limitations of the study, including the small number of bipolar subjects studied. It is difficult to study medication-free bipolar subjects, and a major strength of this study compared to previous studies was that all subjects were medication free for at least 2 months prior to inclusion in the study, with a mean medicationfree period of nearly 50 months. Therefore, the results of the study are much more likely to be reflective of specific lithium effects on peripheral gene expression. The small number of subjects in the BPM and BPD subgroups may have made it more difficult to identify differences between the effects of lithium in the two states as well as the correlation with clinical response. Therefore, a larger number of subjects needs to be studied in future studies.
As mentioned above, a limitation of using peripheral lymphocyte gene expression is that it may not be fully reflective of the CNS $[14,15]$. As brain tissue samples cannot be obtained in living patients, peripheral gene expression may be the best way to study the putative CNS effects of lithium, and could potentially serve as a biomarker of response. In the future, peripheral gene expression studies could be combined with brain imaging studies to further correlate gene expression changes with physiological changes in brain circuits during lithium treatment.

We did not find any differences between bipolar and healthy subjects at baseline, at week 2 or week 8 . Given the variability among subjects, between-group differences at baseline are generally more difficult to identify at significant levels than within-group treatment effect changes. Future studies with a larger number of subjects need to be conducted to identify such changes.

In conclusion, lithium monotherapy treatment in medication-free $\mathrm{BD}$ subjects was associated with changes in transcripts that appeared to plateau by the end of the 2nd week of treatment. Genes and pathways related to immune function, signal transduction, and regulatory molecules were altered. Future studies need to be conducted with a larger number of subjects as well as in conjunction with other physiological phenotypic markers of treatment response such as brain imaging measures.

\section{Acknowledgement}

This project was funded by the NIMH to A.A. (R01MH075025).

\section{Disclosure Statement}

None of the authors have any financial conflicts to declare which are relevant to the conduct of this study.

\section{References}

1 Manji HK, Moore GJ, Chen G: Lithium at 50: have the neuroprotective effects of this unique cation been overlooked? Biol Psychiatry 1999; 46:929-940.

2 Bezchlibnyk Y, Young LT: The neurobiology of bipolar disorder: focus on signal transduction pathways and the regulation of gene expression. Can J Psychiatry 2002;47:135-148.

3 Manji HK, Lenox RH: Long-term action of lithium: a role for transcriptional and posttranscriptional factors regulated by protein kinase C. Synapse 1994;16:11-28. 
4 Chen G, Hasanat KA, Bebchuk JM, Moore GJ, Glitz D, Manji HK: Regulation of signal transduction pathways and gene expression by mood stabilizers and antidepressants. Psychosom Med 1999;61:599-617.

5 Boer U, Cierny I, Krause D, Heinrich A, Lin H, Mayr G, Hiemke C, Knepel W: Chronic lithium salt treatment reduces CRE/CREBdirected gene transcription and reverses its upregulation by chronic psychosocial stress in transgenic reporter gene mice. Neuropsychopharmacology 2007;33:2407-2415.

6 Chetcuti A, Adams LJ, Mitchell PB, Schofield PR: Microarray gene expression profiling of mouse brain mRNA in a model of lithium treatment. Psychiatr Genet 2008;18:64-72.

7 McQuillin A, Rizig M, Gurling HMD: A microarray gene expression study of the molecular pharmacology of lithium carbonate on mouse brain mRNA to understand the neurobiology of mood stabilization and treatment of bipolar affective disorder. Pharmacogenet Genomics 2007;17:605-617.

8 Bosetti F, Seemann R, Bell JM, Zahorchak R, Friedman E, Rapoport SI, Manickam P: Analysis of gene expression with cDNA microarrays in rat brain after 7 and 42 days of oral lithium administration. Brain Res Bull 2002; 57:205-209.

9 Seelan RS, Khalyfa A, Lakshmanan J, Casanova MF, Parthasarathy RN: Deciphering the lithium transcriptome: microarray profiling of lithium-modulated gene expression in human neuronal cells. Neuroscience 2008 ; 151 : 1184-1197.

10 Sun X, Young LT, Wang J-F, Grof P, Turecki G, Rouleau GA, Alda M: Identification of lithium-regulated genes in cultured lymphoblasts of lithium responsive subjects with bipolar disorder. Neuropsychopharmacology 2004;29:799-804.

11 Croce N, Mathé AA, Gelfo F, Caltagirone C, Bernardini S, Angelucci F: Effects of lithium and valproic acid on BDNF protein and gene expression in an in vitro human neuron-like model of degeneration. J Psychopharmacol 2014;28:964-972.

12 Squassina A, Costa M, Congiu D, Manchia M, Angius A, Deiana V, Ardau R, Chillotti C, Severino G, Calza S, Del Zompo M: Insulinlike growth factor 1 (IGF-1) expression is upregulated in lymphoblastoid cell lines of lithium responsive bipolar disorder patients. Pharmacol Res 2013;73:1-7.
13 Sugawara H, Sakamoto K, Harada T, Ishigooka J: Predictors of efficacy in lithium augmentation for treatment-resistant depression. J Affect Disord 2010;125:165-168.

14 Gladkevich A, Kauffman HF, Korf J: Lymphocytes as a neural probe: potential for studying psychiatric disorders. Prog Neuropsychopharmacol Biol Psychiatry 2004;28: 559-576.

15 Sullivan PF, Fan C, Perou CM: Evaluating the comparability of gene expression in blood and brain. Am J Med Genet B Neuropsychiatr Genet 2006;141B:261-268.

16 Lowthert L, Leffert J, Lin A, Umlauf S, Maloney K, Muralidharan A, Lorberg B, Mane S, Zhao H, Sinha R, Bhagwagar Z, Beech R: Increased ratio of anti-apoptotic to pro-apoptotic Bcl2 gene-family members in lithiumresponders one month after treatment initiation. Biol Mood Anxiety Disord 2012;2:15.

17 Beech RD, Leffert JJ, Lin A, Sylvia LG, Umlauf S, Mane S, Zhao H, Bowden C, Calabrese JR, Friedman ES, Ketter TA, Iosifescu DV, ReillyHarrington NA, Ostacher M, Thase ME, Nierenberg A: Gene-expression differences in peripheral blood between lithium responders and non-responders in the Lithium Treatment-Moderate dose Use Study (LiTMUS). Pharmacogenomics J 2014;14:182-191.

18 Nurnberger JI Jr, Blehar MC, Kaufmann CA, York-Cooler C, Simpson SG, Harkavy-Friedman J, Severe JB, Malaspina D, Reich T: Diagnostic interview for genetic studies. Rationale, unique features, and training. NIMH Genetics Initiative. Arch Gen Psychiatry 1994;51: 849-859; discussion 63-64.

19 American Psychiatric Association: Diagnostic and Statistical Manual 4th Edition - Text Revision (DSM-IV-TR). Washington, American Psychiatric Association, 2000.

20 Young RC, Biggs JT, Ziegler VE, Meyer DA: A rating scale for mania: reliability, validity and sensitivity. Br J Psychiatry 1978;133:429435.

21 Busner J, Targum SD: The Clinical Global Impressions Scale: applying a research tool in clinical practice. Psychiatry (Edgmont) 2007; 4:28-37.

22 Sheehan DV, Lecrubier Y, Sheehan KH, Amorim P, Janavs J, Weiller E, Hergueta T, Baker R, Dunbar GC: The Mini-International Neuropsychiatric Interview (M.I.N.I.): the development and validation of a structured diagnostic psychiatric interview for DSM-IV and ICD-10. J Clin Psychiatry 1998;59(suppl 20):22-33; quiz 4-57.
23 McClintick JN, Edenberg HJ: Effects of filtering by present call on analysis of microarray experiments. BMC Bioinformatics 2006;7:49.

24 Storey JD, Tibshirani R: Statistical significance for genomewide studies. Proc Natl Acad Sci USA 2003;100:9440-9445.

25 Setiawan E, Wilson AA, Mizrahi R, et al: Role of translocator protein density, a marker of neuroinflammation, in the brain during major depressive episodes. JAMA Psychiatry 2015;72:268-275.

26 Becking K, Boschloo L, Vogelzangs N, Haarman BCM, Riemersma-van der Lek R, Penninx BWJH, Schoevers RA: The association between immune activation and manic symptoms in patients with a depressive disorder. Transl Psychiatry 2013;3:e314.

27 Haarman BCM, Riemersma-Van der Lek RF, de Groot JC, Ruhé HG, Klein HC, Zandstra TE, Burger H, Schoevers RA, de Vries EFJ, Drexhage HA, Nolen WA, Doorduin J: Neuroinflammation in bipolar disorder - A [11C]-(R)-PK11195 positron emission tomography study. Brain Behav Immun 2014; 40:219-225.

28 Maletic V, Raison C: Integrated neurobiology of bipolar disorder. Front Psychiatry 2014;5: 98.

29 Bosetti F, Rintala J, Seemann R, Rosenberger TA, Contreras MA, Rapoport SI, Chang MC: Chronic lithium downregulates cyclooxygenase- 2 activity and prostaglandin $\mathrm{E}(2)$ concentration in rat brain. Mol Psychiatry 2002;7: 845-850.

30 Tkachev D, Mimmack ML, Ryan MM, Wayland $\mathrm{M}$, Freeman $\mathrm{T}$, Jones $\mathrm{PB}$, Starkey $\mathrm{M}$, Webster MJ, Yolken RH, Bahn S: Oligodendrocyte dysfunction in schizophrenia and bipolar disorder. Lancet 2003;362:798-805.

31 Lian S, Shi R, Bai T, Liu Y, Miao W, Wang H, Liu X, Fan Y: Anti-miRNA-23a oligonucleotide suppresses glioma cells growth by targeting apoptotic protease activating factor-1. Curr Pharm Des 2013;19:6382-6389.

32 de Sousa RT, Zarate Jr CA, Zanetti MV, Costa AC, Talib LL, Gattaz WF, Machado-Vieira R: Oxidative stress in early stage bipolar disorder and the association with response to lithium. J Psychiatr Res 2014;50:36-41. 